\title{
Minimal Composite Dynamics versus Axion Origin of the Diphoton excess
}

\author{
Emiliano Molinaro, Francesco Sannino, and Natascia Vignaroli \\ $C P^{3}$-Origins and the Danish IAS, University of Southern Denmark, Campusvej 55, DK-5230 Odense M, Denmark
}

\begin{abstract}
ATLAS and CMS observe deviations from the expected background in the diphoton invariant mass searches of new resonances around $750 \mathrm{GeV}$. We show that a simple realization in terms of a new pseudoscalar state can accommodate the observations. The model leads to further footprints that can be soon observed. The new state can be interpreted both as an axion or as a highly natural composite state arising from minimal models of dynamical electroweak symmetry breaking. We further show how to disentangle the two scenarios. Beyond the possible explanation of the diphoton excess the results show that it is possible to directly test and constrain composite dynamics via processes stemming from its distinctive topological sector.
\end{abstract}

The ATLAS and CMS [1, 2, find local excesses respectively of $3.6 \sigma$ and $2.6 \sigma$ for a resonance in diphoton searches with invariant mass spectrum around $750 \mathrm{GeV}$. This leads to a global significance of $2 \sigma$ in ATLAS and $1.2 \sigma$ in CMS.

The ATLAS and CMS results in the $a \rightarrow 2 \gamma$ channel suggest a reconstructed mass of around $750 \mathrm{GeV}$ and a cross-section $\sigma(p p \rightarrow a \rightarrow 2 \gamma)$ of the order of $6 \mathrm{fb}$. Here $a$ denotes a new intermediate massive state.

We assume $a$ to be a neutral spin zero particle and employ a minimal description in terms of an effective field theory, and study the resulting phenomenology in the narrow width approximation. After introducing the relevant effective operators we show how they can emerge within two calculable extensions of the Standard Model (SM) featuring either a new elementary axion-like or a composite $\eta^{\prime}$-like state. We further assume both states to couple to a new colored vectorlike fermion $T$. The underlying realisations allow us to make further predictions and relate some of the effective couplings. The models encompass all the needed ingredients to describe the signal channels and relevant constraints. We will also provide distinctive signatures aimed at disentangling the composite nature from the elementary one.

We then make contact with time-honoured models of minimal composite dynamics 3 6. Weinberg and Susskind's minimal models of dynamical electroweak (EW) symmetry breaking [3, 4, are based on QCD-like dynamics and are at odds with experiments. Within this early model realisations one finds the pioneering work of Di Vecchia and Veneziano 7 that long ago envisioned a scenario similar to the one presented here. Modern incarnations that are still minimal but employ non-QCD like dynamics are phenomenologically viable 8 10. Complementary signal channels for spin-one resonances have been investigated in more complete model implementations, e.g. in [11, that can even explain the $2-\mathrm{TeV}$ diboson excess 12. The general features, regarding resonance mass, cross-section and decay patterns are very much in line with models of weak scale compositeness [8 11].

Therefore the minimal weak-scale composite paradigm, besides solving the hierarchy problem:

- explains the diphoton excess,
- naturally accommodates the $750 \mathrm{GeV}$ mass,

- predicts and relates new processes,

- can be disentangled from other less natural models.

Amusingly, the best fit value for the decay constant of the new state is highly compatible with the one needed to break the EW symmetry dynamically.

Assuming the existence of a new pseudoscalar state $a$ the CP conserving effective operators linking the spinzero resonance with the SM fermions are

$$
\mathcal{L}_{\mathrm{qq}}^{a}=-\sum_{i j} i a \bar{q}_{i} Y_{i j}^{a} \gamma_{5} q_{j}
$$

where $i, j$ runs over all flavors. The effective operators linking our states to SM gauge bosons are

$$
\mathcal{L}_{V \widetilde{V}}^{a}=-\sum_{V_{1} \widetilde{V}_{2}} \frac{g_{V_{1} \widetilde{V}_{2}}}{8} a V_{1} \widetilde{V}_{2}
$$

with

$$
\begin{aligned}
\sum_{V_{1} \widetilde{V}_{2}} g_{V_{1} \widetilde{V}_{2}} V_{1} \widetilde{V}_{2} & =g_{G \widetilde{G}} \operatorname{Tr}\left[G_{\mu \nu} \widetilde{G}^{\mu \nu}\right]+g_{A \widetilde{A}} A_{\mu \nu} \widetilde{A}^{\mu \nu} \\
& +g_{Z \widetilde{Z}} Z_{\mu \nu} \widetilde{Z}^{\mu \nu}+2 g_{W \widetilde{W}} W_{\mu \nu}^{+} \widetilde{W}^{-\mu \nu} \\
& +2 g_{Z \widetilde{A}} Z_{\mu \nu} \widetilde{A}^{\mu \nu}
\end{aligned}
$$

and $\widetilde{V}^{\mu \nu}=\epsilon^{\mu \nu \rho \sigma} V_{\rho \sigma}$. Depending on the underlying realisation some of these couplings might either vanish, develop hierarchies and/or be related to each other. In the following we will consider the case in which the effective couplings in (1) are negligible.

\section{Axion Realisation}

We add to the SM the terms:

$$
\begin{aligned}
\Delta \mathcal{L} & =\frac{1}{2}\left(\partial_{\mu} a \partial^{\mu} a-m_{a}^{2} a^{2}\right)+i \bar{T} \gamma_{\mu} D^{\mu} T-i y_{T} \frac{m_{T}}{f_{a}} a \bar{T} \gamma_{5} T \\
& -m_{T} \bar{T} T+\Delta_{t-T}^{\mathrm{mix}}
\end{aligned}
$$

where $T$ is an $\mathrm{SU}(2)$ weak singlet vectorlike quark in a given representation of color interactions. The new Yukawa interaction strength is controlled by $y_{T}$ and the $a$ 
decay constant $f_{a}$. The mixing mass-term operator $\Delta_{t-T}^{\operatorname{mix}}$ between the top and the new colored state vanishes unless the representation of $T$ is the fundamental of color and/or the hypercharge matches. The action above can represent that of an axion-like state. We will see later that a more natural interpretation emerges when this state is viewed as a composite one, provided new operators are added stemming from its topological sector.

$T$ loops, in the fundamental representation of color, generate the following effective couplings

$g_{G \widetilde{G}}^{T}=y_{T} \frac{\alpha_{S}}{2 \pi f_{a}} F\left(\frac{m_{a}^{2}}{4 m_{T}^{2}}\right), g_{A \widetilde{A}}^{T}=y_{T} \frac{4}{3} \frac{\alpha_{\mathrm{em}}}{\pi f_{a}} F\left(\frac{m_{a}^{2}}{4 m_{T}^{2}}\right)$,

$g_{A \widetilde{Z}}^{T}=\tan \left(\theta_{W}\right) g_{A \widetilde{A}}, \quad g_{Z \widetilde{Z}}^{T}=\tan ^{2}\left(\theta_{W}\right) g_{A \widetilde{A}}$,

where $\theta_{W}$ is the weak mixing angle and, in the approximation $m_{a}<2 m_{T}$, we have $F(x)=\arcsin (\sqrt{x}) / x \approx$ $1+x / 3+8 x^{2} / 45+4 x^{3} / 35$. From the model we arrive at the following relevant partial decay rates

$$
\begin{aligned}
\Gamma(a \rightarrow g g) & =\frac{m_{a}^{3}}{8 \pi}\left(g_{G \widetilde{G}}^{T}\right)^{2}, \\
\Gamma(a \rightarrow \gamma \gamma) & =\frac{m_{a}^{3}}{64 \pi}\left(g_{A \widetilde{A}}^{T}\right)^{2}, \\
\Gamma(a \rightarrow \gamma Z) & =\frac{m_{a}^{3}}{32 \pi}\left(g_{A \widetilde{Z}}^{T}\right)^{2}\left(1-\frac{m_{Z}^{2}}{m_{a}^{2}}\right)^{3}, \\
\Gamma(a \rightarrow Z Z) & =\frac{m_{a}^{3}}{64 \pi}\left(g_{Z \widetilde{Z}}^{T}\right)^{2}\left(1-\frac{4 m_{Z}^{2}}{m_{a}^{2}}\right)^{3 / 2},
\end{aligned}
$$

and branching ratios

$$
\begin{aligned}
B(a \rightarrow \gamma \gamma) & \approx 0.0063 \\
B(a \rightarrow \gamma Z) & \approx 0.0037 \\
B(a \rightarrow Z Z) & \approx 0.00055
\end{aligned}
$$

From the above it is clear that the branching ratio into two photons dominates with respect to the other EW channels. Furthermore, while the partial widths depend directly on $f_{a} / y_{T}$ and $m_{a}$ the branching ratios depend only on $\alpha_{S}$ and weak coupling constants.

We implement our model in MadGraph5_aMC@NLO [13. with the effective couplings in (5) and we calculate the $a$ production cross-section at leading order. The QCD next-to-leading order (NLO) corrections are calculated using the model provided in 14. For $m_{a} \simeq 750$ $\mathrm{GeV}$ we obtain a NLO $K$-factor: $K^{N L O} \simeq 2.6$.

Fig. 1 shows the production cross-section of the pseudoscalar $a$ at the LHC with $\sqrt{s}=13 \mathrm{TeV}$ as function of $m_{a}$ for the specific $f_{a} / y_{T}$ value which reproduces the ATLAS excess, and for a $1 \mathrm{TeV}$ vectorlike quark $T$.

A fit to the ATLAS excess, in the narrow-widthapproximation, gives the value $\sigma(p p \rightarrow a \rightarrow 2 \gamma)=$ $(6 \pm 3) \mathrm{fb}$. Remarkably this value is reproduced by our model for an $f_{a}$ scale

$$
f_{a} /\left|y_{T}\right|=245_{-45}^{+100} \mathrm{GeV},
$$

with $m_{T}=1 \mathrm{TeV}$. We find a very mild dependence of our results on the $T$ mass. A lower limit on $m_{T}$ of the order

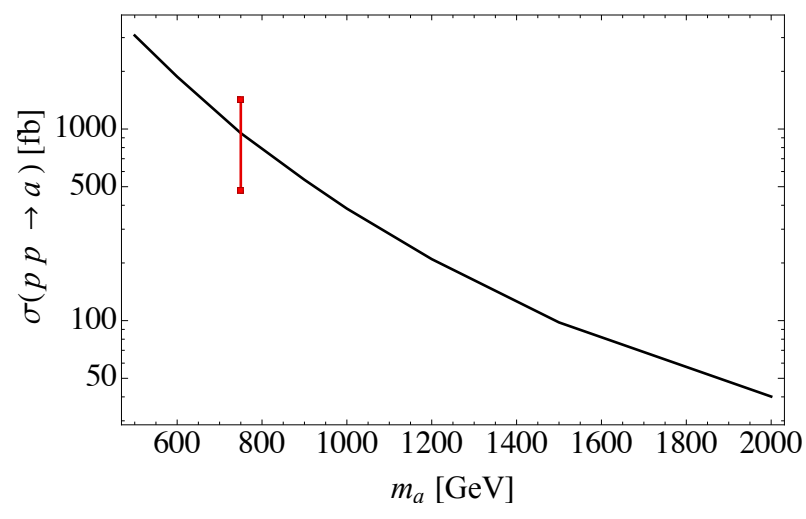

FIG. 1. Production cross-section of $a$ at the $13 \mathrm{TeV}$ LHC as function of the pseudoscalar mass, for the value $f_{a} / y_{T}=245$ $\mathrm{GeV}$ reproducing the ATLAS excess and $m_{T}=1 \mathrm{TeV}$. The vertical bar at $750 \mathrm{GeV}$ shows the $\pm 1 \sigma$ deviation from the central value of the excess. The cross-section scales as $y_{T}^{2} / f_{a}^{2}$.

of $800 \mathrm{GeV}$ is placed by the run-1 LHC results [15, 16]. The vectorlike quark coupling to $a, y_{T} m_{T} / f_{a}$, reaches the non-perturbative regime for $m_{T} \gtrsim 1 / y_{T} \mathrm{TeV}$.

On top of the presence of the vectorlike $T$, possibly within the reach of the current LHC run [17, the model predicts a significant $a$ decay branching ratio to $Z \gamma$. The corresponding signal with $Z$ decaying to leptons could be observed when the experiments will have collected roughly $100 \mathrm{fb}^{-1}$. This would correspond to a number of expected signal events comparable with those in the $\gamma \gamma$ excess. The narrow $a$ resonance can also be detected via di-jets. However, the sensitivity of the $8 \mathrm{TeV}$ searches [18, 19] in this channel is low, due to the overwhelming QCD background at the relatively low di-jet mass spectrum near $750 \mathrm{GeV}$. In fact, the CMS search 19 places an upper limit on the di-jet cross-section times acceptance of about $1.8 \mathrm{pb}$ for a resonance at $750 \mathrm{GeV}$, which is an order of magnitude above the value we obtain for the $a$ pseudoscalar, that is $\sim 70 \mathrm{fb}$, assuming an acceptance of 0.6. The run-1 LHC searches in the $\gamma \gamma$ channel at $\sqrt{s}=8 \mathrm{TeV}$ [20, 21] are also compatible with our explanation of the diphoton excess at $13 \mathrm{TeV}$. The $95 \%$ C.L. upper limit on the $\gamma \gamma$ cross-section at the invariant mass $m_{\gamma \gamma} \sim 750 \mathrm{GeV}$ is of about $1.5 \mathrm{fb}$, while the axion model predicts about $0.7 \mathrm{fb}$. The present explanation of the ATLAS and CMS diphoton excesses, therefore, passes all of the current experimental tests.

\section{Minimal Composite Dynamics}

We move now to the interpretation of the new state within minimal (near-conformal) models of dynamical EW symmetry breaking [8, 9, 22, 27 .

In particular the new heavy resonance can be identified, in composite models at the EW scale, with the singlet $\eta^{\prime}$ state that we rename here $a$. This requires a new strongly coupled sector featuring $N_{T}^{F}$ Dirac flavors 
transforming under the representation $R$ of a new gauge sector $S U\left(N_{T}\right)$ with $N_{T}$ the new color.

Assuming that the number of flavors is barely outside the conformal window 26, the new gauge sector will generate chiral symmetry breaking with confining scale $\Lambda_{T}$ and technipion decay constant $F_{T}$. At energies below $\Lambda_{T}$ the relevant degrees of freedom, needed for the present analysis, are encoded in the unitary matrix

$$
U=e^{i \Phi / F_{T}}=\exp \left[\frac{i}{F_{T}}(a+\vec{\tau} \cdot \vec{\pi})\right]
$$

where $\vec{\tau} \equiv\left(\tau_{1}, \tau_{2}, \tau_{3}\right)$ are the standard Pauli matrices, whereas $a$ and $\vec{\pi} \equiv\left(\pi^{1}, \pi^{2} \pi^{3}\right)$ are, respectively, the singlet and isotriplet pseudoscalar resonances. For simplicity we assume $N_{T}^{F}=2$, but it is straightforward to generalise this to a different number of flavors. The associated quantum global symmetry is $S U(2)_{L} \times S U(2)_{R}$. Although a larger symmetry group can be considered for a possible realisation within composite Goldstone Higgs dynamics we focus here on minimal composite realisations 11 Of course, we should also include a Higgs-like state. Depending on the explicit underlying dynamics, it might emerge either as the lightest dilaton-like state stemming from a near conformal theory [9, 28, or, if the symmetry is opportunely enhanced, as pseudo Goldstone Boson [5, 6. Furthermore near-conformality ${ }^{2}$ alleviates tension with EW precision measurements 22] and flavor changing neutral currents constraints [40].

The effective action generating the relevant $a$ interaction is:

$$
\Gamma=\int d^{4} x\left(\mathcal{L}_{0}+\mathcal{L}_{m_{a}}\right)+\Gamma_{W Z W} .
$$

Each term in (13) can be expressed in terms of $U$, the Maurer-Cartan one-forms

$$
\alpha=\left(\partial_{\mu} U\right) U^{-1} d x^{\mu} \equiv(d U) U^{-1}, \quad \beta=U^{-1} \alpha U
$$

and additional "left" and "right" one-forms, $A_{L}=$ $A_{L}^{\mu} d x_{\mu}$ and $A_{R}=A_{R}^{\mu} d x_{\mu}$, respectively, with

$A_{L}^{\mu}=g_{Y}\left(Q-\frac{1}{2} \tau_{3}\right) B_{\mu}+\frac{1}{2} g_{W} \vec{\tau} \cdot \vec{W}^{\mu}, \quad A_{R}^{\mu}=g_{Y} Q B^{\mu}$,

where $Q$ denotes the electric charge matrix of the fundamental technifermions.

\footnotetext{
1 Here the underlying dynamics does not carry color. This guarantees minimality, meaning that no new colored technihadron states are present in the spectrum. Uncolored technifermions are also preferred by precision EW observables.

2 Analytical [8, 26, 29] and numerical efforts [30, 38 have been dedicated to determine whether fermionic gauge theories display large distance conformality. For the sextet model lattice results suggest that the theory is either very near-conformal or conformal. In the latter case interactions responsible for giving masses to the SM fermions can modify the conformal-boundary inducing an ideal near-conformal behaviour [39].
}

The relevant Lagrangian terms are

$$
\begin{aligned}
\mathcal{L}_{0} & =\mathcal{L}_{\text {kin }}+\frac{F_{T}^{2}}{2} \operatorname{Tr}\left[\partial_{\mu} \xi^{\dagger} \partial^{\mu} \xi\right]-\frac{F_{T}^{2}}{2} \operatorname{Tr}\left[\xi^{\dagger} \partial_{\mu} \xi \partial^{\mu} \xi \xi^{\dagger}\right] \\
& +\frac{F_{T}^{2}}{4} \operatorname{Tr}\left[A_{L}^{\mu} A_{L \mu}+A_{R}^{\mu} A_{R \mu}\right]-\frac{F_{T}^{2}}{2} \operatorname{Tr}\left[A_{L}^{\mu} U A_{R \mu} U^{\dagger}\right] \\
& -\frac{i F_{T}^{2}}{2} \operatorname{Tr}\left[\partial_{\mu} \xi \xi^{\dagger} A_{L}^{\mu}-\xi^{\dagger} \partial_{\mu} \xi A_{R}^{\mu}\right] \\
& -\frac{i F_{T}^{2}}{2} \operatorname{Tr}\left[\xi \partial_{\mu} \xi U^{\dagger} A_{L}^{\mu}-\partial_{\mu} \xi \xi^{\dagger} A_{R}^{\mu} U^{\dagger}\right]
\end{aligned}
$$

where $\xi \equiv U^{1 / 2}$ and $\mathcal{L}_{\text {kin }}$ is the standard kinetic term for the vector fields. The second term in 13 comes from a quantum anomaly and it provides a mass term for the singlet $a$. It reads

$$
\mathcal{L}_{m_{a}}=\frac{\kappa F_{T}^{2}}{8 N_{T}} \operatorname{Tr}\left[\ln U-\ln U^{\dagger}\right]^{2}
$$

and $\kappa$ is connected to the mass of $\eta_{0}\left(m_{\eta_{0}}=849 \mathrm{MeV}\right),{ }^{3}$

$$
\kappa=\frac{1}{6} \frac{F_{T}^{2}}{f_{\pi}^{2}} \frac{9}{N_{T}} m_{\eta_{0}}^{2} .
$$

Then, the mass of $a$ (taking $f_{\pi}=92 \mathrm{MeV}$ ), for technifermions in the fundamental representation, is

$$
m_{a}=\sqrt{\frac{2}{3}} \frac{F_{T}}{f_{\pi}} \frac{3}{N_{T}} m_{\eta_{0}} \approx \frac{6}{N_{T}} \mathrm{TeV} .
$$

Thus, we have that a $750 \mathrm{GeV} a$-state naturally emerges in this scenario for $N_{T}=6$ and 8 . Similar values of $m_{a}$ could emerge from near conformal field theories with smaller $N_{T}$ 8, 42 .

As for the relevant terms arising from the gauged WessZumino-Witten operator [7, 41, 43, 44] we have:

$$
\begin{aligned}
& \Gamma_{W Z W}\left[U, A_{L}, A_{R}\right]=\Gamma_{W Z}[U] \\
& -5 C \int_{M^{4}} \operatorname{Tr}\left[\left(d A_{L} A_{L}+A_{L} d A_{L}\right) \alpha+\left(d A_{R} A_{R}+A_{R} d A_{R}\right) \beta\right] \\
& +5 C \int_{M^{4}} \operatorname{Tr}\left[d A_{L} d U A_{R} U^{-1}-d A_{R} d U^{-1} A_{L} U\right]+\cdots, \quad(20)
\end{aligned}
$$

where $C=-i d(R) /\left(240 \pi^{2}\right), d(R)$ is the dimension of the technifermion representation. For the fundamental representation $d$ (Fund) $=N_{T}$. Here $F_{L}$ and $F_{R}$ are twoforms defined as $F_{L}=d A_{L}-i A_{L}^{2}$ and $F_{R}=d A_{R}-$ $i A_{R}^{2}$. The Wess-Zumino effective action is $\Gamma_{W Z}[U]=$ $C \int_{M^{5}} \operatorname{Tr}\left[\alpha^{5}\right]$. From 20 we have extra contributions to the effective couplings to the gauge bosons given in

\footnotetext{
${ }^{3}$ Here $\eta_{0}$ indicates the QCD SU(3) flavor singlet state in the chiral limit, with 41 ]

$$
m_{\eta_{0}}^{2}=m_{\eta^{\prime}}^{2}+m_{\eta}^{2}-2 m_{K}^{2}
$$


(2) that alter the predictions with respect to the axionlike scenario. These extra contributions are:

$$
\begin{gathered}
g_{A \widetilde{A}}^{\mathrm{comp}}=\left(1+y^{2}\right) \frac{e^{2}}{F_{T}} \frac{d(R)}{8 \pi^{2}}, \\
g_{A \widetilde{Z}}^{\mathrm{comp}}=\frac{1-2\left(1+y^{2}\right) s_{W}^{2}}{2 c_{W} s_{W}} \frac{e^{2}}{F_{T}} \frac{d(R)}{8 \pi^{2}}, \\
g_{Z \widetilde{Z}}^{\mathrm{comp}}=\frac{e^{2}}{F_{T}} \frac{1-3 s_{W}^{2}+3\left(1+y^{2}\right) s_{W}^{4}}{3 c_{W}^{2}} \frac{d(R)}{8 \pi^{2}}, \\
g_{W \widetilde{W}}^{\text {comp }}=\frac{e^{2}}{F_{T}} \frac{1}{s_{W}^{2}} \frac{d(R)}{24 \pi^{2}},
\end{gathered}
$$

$y$ being the hypercharge of the (left-handed) fundamental technifermions [7] with the normalisation $Y\left(Q_{L}\right)=y / 2$ and $Y\left(U_{R} / D_{R}\right)=(y \pm 1) / 2$ and $Q=Y+\tau_{3} / 2$. Here $Q_{L}$ is the techniquark $S U(2)_{L}$ transforming doublet and $Q_{R}=\left(U_{R}, D_{R}\right)$ the singlets 4 .

To compare with the elementary scenario, we added the same coupling in (4) between the composite $a$ and the new fermion $T$. It could be generated via instantons from an extended gauge dynamics (EGD). Near the EW scale the EGD would lead to the effective 6 -fermion operator $\operatorname{det}\left(\bar{Q}_{L} Q_{R}\right) \bar{T}_{L} T_{R}+$ h.c. suppressed by $\Lambda_{\mathrm{EGD}}^{5}$. Since the UV scale $\Lambda_{\mathrm{EGD}}$ is expected to be larger than the EW scale, this operator would typically lead to small values of $y_{T}$.

The effective couplings of $a$ to the EW gauge bosons are therefore given by $g_{V \widetilde{V}}=g_{V \widetilde{V}}^{c o m p}+g_{V \tilde{V}}^{T}$, where $g_{V \widetilde{V}}^{T}$ are reported in (5). We can now calculate the region of the parameter space which allows to reproduce the diphoton excess in the $\eta^{\prime}$-like scenario introduced above. We assume $F_{T}=246 \mathrm{GeV}$ and $d($ Fund $)=N_{T}=6$ in such a way to naturally reproduce the pseudoscalar mass $m_{a} \approx 750 \mathrm{GeV}$, see eq. 19 . We can also have $d(R)=6$ when the technifermions are in a symmetric of $S U\left(N_{T}\right)$ with $N_{T}=3$. Precision observables were studied in [9].

We show in the left panel of Fig. $2 y_{T}$ versus $y$ regions which reproduce the diphoton excess within $\pm 1 \sigma$. The results do not change significantly by varying $d(R)$. The $\left(y_{T}, y\right)$ solutions, for example, that we obtain for $d(R)=4$ overlap with those for $d(R)=6$. We have three different regions of $\left(y_{T}, y\right)$ parameters for which the diphoton excess can be reproduced, as shown in the left panel of Fig. 2. Region 1 corresponds to two solutions with almost identical small values of $\left|y_{T}\right|$, in

4 The possible presence of the Witten 45 and/or SM gauge anomalies depends on the underlying technifermion representation and hypercharge assignment. If present, one can cancel them by adding new lepton-like heavy fermions [7, 9]. Direct limits on stable singly-charged SM-like heavy fermions require them to be heavier than about $574 \mathrm{GeV}$ [4]. Since the couplings of the new leptons to SM degrees of freedom depend on unknown dynamics, these limits should be taken cum grano salis. agreement with our naive expectation from EGD. Region 2 and 3 correspond to negative $y_{T}$ values and fall in the large-coupling regime of the a coupling to $T$, $\left|y_{T}\right| m_{T} / F_{T}>\sqrt{4 \pi}$. We include in the calculation of the production cross-section the photon fusion mechanism [47, which gives a contribution larger than 50\% (smaller than 10\%) to the total cross-section for $y \gtrsim 1.2$ $(y \lesssim 0.7)$ 48.

The predictions for the branching ratios of $a$ decaying into the possible different pairs of EW gauge bosons and gluons are reported in the right panel of Fig. 2 and are calculated for the positive $y_{T}$ solutions in the perturbative region 1 . The $\eta^{\prime}$-like explanation of the diphoton excess passes all of the current constraints from the LHC searches for diboson resonances. At $\sqrt{s}=13 \mathrm{TeV}$, for a resonance of $\sim 750 \mathrm{GeV}$, the strongest constraint on the $Z Z(W W)$ channel is placed by the ATLAS search in 49 ([50]) which gives an upper limit of about $250(250) \mathrm{fb}$ on $\sigma(p p \rightarrow a \rightarrow Z Z(W W))$, while for our $\eta^{\prime}$ we have at most $\sim 4(20) \mathrm{fb}$. The run-1 searches at $\sqrt{s}=8 \mathrm{TeV}$ give an upper limit on the resonance cross-section of about $10 \mathrm{fb}$ in the $Z Z$ channel [51, to be compared with at most $\sim 1 \mathrm{fb}$ for our $\eta^{\prime}$, and of about $40 \mathrm{fb}$ in the $W W$ channel [52], where we have at most $\sim 4 \mathrm{fb}$. Finally, the ATLAS search on the $Z \gamma$ channel 53 gives an upper limit $\sigma(p p \rightarrow a \rightarrow Z \gamma) \lesssim 4 \mathrm{fb}$, which is fulfilled in our scenario, predicting a cross-section of at most $\sim 1 \mathrm{fb}$.

The di-jet channel from the $a \rightarrow g g$ decay, which is the dominant decay mode for the axion-like scenario discussed in the first part of this work is instead suppressed for the $\eta^{\prime}$-like state. The $W W$ channel, which is absent for the axion-like particle may have a relevant branching ratio which is enhanced compared to $\gamma \gamma$ for $y \lesssim 1$. The $Z Z$ channel is also enhanced compared to the axion-like scenario, especially for smaller $y$ values. Finally, the $\gamma Z$ channel is enhanced compared to the axion-like case for $y \lesssim 0.5$, whereas it is suppressed for larger $y$ values.

In the present study we have not included the potential effects of a direct SM top coupling to either the elementary or composite $a$ particle. In the elementary case we have checked that, when one includes the coupling to the top $m_{t} / f_{a} \sim 0.7$, the branching ratio in diphoton is too small to explain the excess because the total width of $a$ is dominated by the tree-level decay into SM tops. In the composite case the actual strength of the coupling to the SM top depends on the underlying scenario for the SM fermion mass generation. To provide a direct comparison with the axion case we have considered above scenarios in which the coupling to the SM top is suppressed. However, we have also investigated the case in which this coupling is as big as $m_{t} / F_{T}$. We find that the excess can be reproduced, unlike the axion case, because of the topological terms, with and without the inclusion of the extra $T$ fermion. We also checked that this scenario is consistent with the run-1 LHC experimental bounds on $\bar{t} t$ resonances [54, 55].

Our results show that a highly natural and minimal composite nature of the new potential particle, in terms 

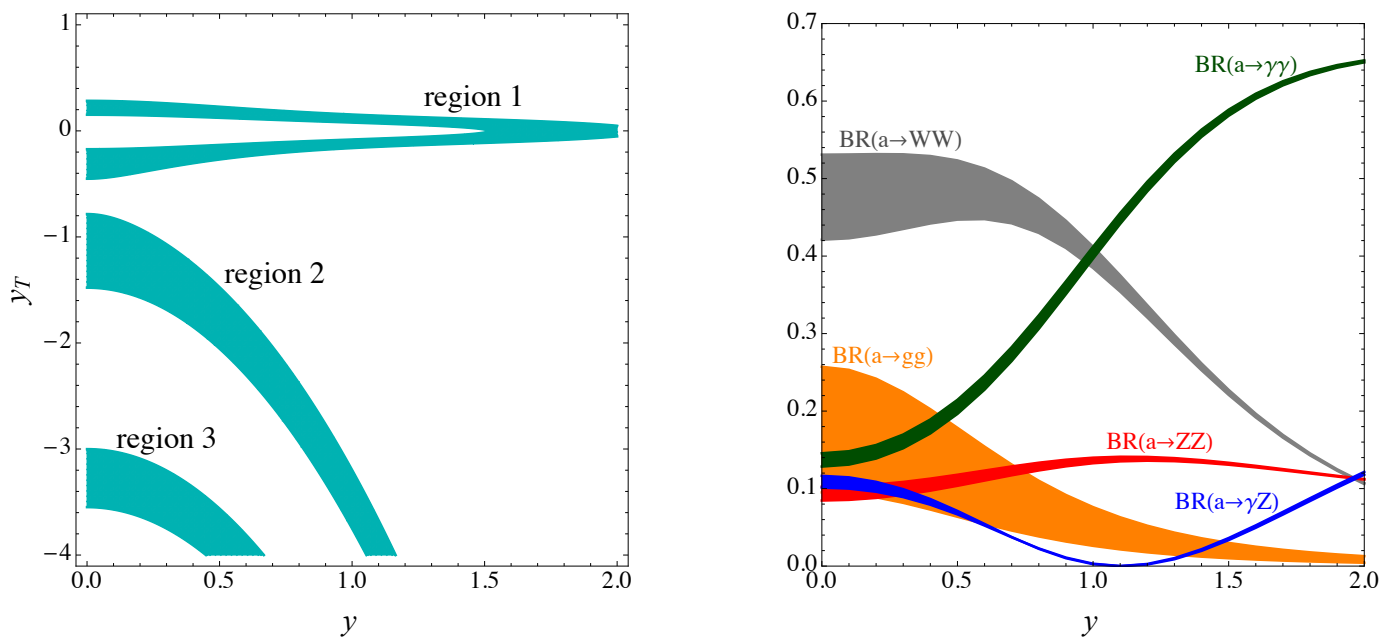

FIG. 2. Left panel: Correlation between $y_{T}$ and the hypercharge $y$ of the fundamental (left-handed) technifermions that allows to explain the diphoton excess in the $\eta^{\prime}$-like scenario. Region 2 and region 3 correspond to large couplings of the $a$ to the vectorlike quark T. Right panel: Branching ratios for the decay of $a$ into a pair of gauge bosons predicted in the $\eta^{\prime}$-like scenario as a function of $y$, calculated for values of $y_{T} \geq 0$ in region 1 . For $y_{T}=0$ the production of the resonance is entirely given by the photon fusion process.

of an $\eta^{\prime}$-like state, decaying into two photons can explain the excess. We have also demonstrated that the underlying axion or minimal composite nature of this state can be disentangled upon discovery and careful analysis of the related decay channels. Furthermore, our analysis is immediately applicable to set relevant constraints on EW scale composite dynamics at run-2 LHC.

Acknowledgments and note added: The $\mathrm{CP}^{3}$-Origins center is partially funded by the Danish National Research Foundation, grant number DNRF90. While this paper was being completed a number of papers appeared [56. 57] that partially overlapped with the present one.
[1] ATLAS-CONF-2015-081

[2] CMS PAS-EXO-15-004

[3] S. Weinberg, Phys. Rev. D 13, 974 (1976). doi:10.1103/PhysRevD.13.974

[4] L. Susskind, Phys. Rev. D 20, 2619 (1979). doi:10.1103/PhysRevD.20.2619

[5] D. B. Kaplan and H. Georgi, Phys. Lett. B 136, 183 (1984). doi:10.1016/0370-2693(84)91177-8

[6] D. B. Kaplan, H. Georgi and S. Dimopoulos, Phys. Lett. B 136, 187 (1984). doi:10.1016/0370-2693(84)91178-X

[7] P. Di Vecchia and G. Veneziano, Phys. Lett. B 95, 247 (1980). doi:10.1016/0370-2693(80)90480-3

[8] F. Sannino and K. Tuominen, Phys. Rev. D 71, 051901 (2005) doi:10.1103/PhysRevD.71.051901 hep$\mathrm{ph} / 0405209$.

[9] D. D. Dietrich, F. Sannino and K. Tuominen, Phys. Rev. D 72, 055001 (2005) doi:10.1103/PhysRevD.72.055001 hep-ph/0505059.

[10] T. A. Ryttov and F. Sannino, Phys. Rev. D 78, 115010 (2008) doi:10.1103/PhysRevD.78.115010 arXiv:0809.0713 [hep-ph]].

[11] A. Belyaev, R. Foadi, M. T. Frandsen, M. Jarvinen, F. Sannino and A. Pukhov, Phys. Rev. D 79, 035006 (2009) doi:10.1103/PhysRevD.79.035006 arXiv:0809.0793 [hep-ph]].

[12] D. B. Franzosi, M. T. Frandsen and F. San- nino, Phys. Rev. D 92, $115005 \quad$ (2015) doi:10.1103/PhysRevD.92.115005 arXiv:1506.04392 [hep-ph]].

[13] J. Alwall, M. Herquet, F. Maltoni, O. Mattelaer and T. Stelzer, JHEP 1106, 128 (2011) doi:10.1007/JHEP06(2011)128 arXiv:1106.0522 [hep$\mathrm{ph}]$.

[14] F. Demartin, F. Maltoni, K. Mawatari, B. Page and M. Zaro, Eur. Phys. J. C 74, no. 9, 3065 (2014) doi:10.1140/epjc/s10052-014-3065-2 arXiv:1407.5089 [hep-ph]].

[15] CMS Collaboration [CMS Collaboration], CMS-PASB2G-12-017.

[16] The ATLAS collaboration [ATLAS Collaboration], ATLAS-CONF-2015-012.

[17] N. Vignaroli, Phys. Rev. D 86, 075017 (2012) doi:10.1103/PhysRevD.86.075017 arXiv:1207.0830 [hep$\mathrm{ph}]$.

[18] G. Aad et al. [ATLAS Collaboration], Phys. Rev. D 91, no. 5, 052007 (2015) doi:10.1103/PhysRevD.91.052007 arXiv:1407.1376 [hep-ex]].

[19] CMS Collaboration [CMS Collaboration], CMS-PASEXO-14-005.

[20] G. Aad et al. [ATLAS Collaboration], Phys. Rev. D 92, no. 3, 032004 (2015) doi:10.1103/PhysRevD.92.032004 arXiv:1504.05511 [hep-ex]]. 
[21] V. Khachatryan et al. [CMS Collaboration], Phys. Lett. B 750, 494 (2015) doi:10.1016/j.physletb.2015.09.062 arXiv:1506.02301 [hep-ex]].

[22] T. Appelquist and F. Sannino, Phys. Rev. D 59, 067702 (1999) doi:10.1103/PhysRevD.59.067702 hep$\mathrm{ph} / 9806409$.

[23] T. Appelquist, P. S. Rodrigues da Silva and F. Sannino, Phys. Rev. D 60, 116007 (1999) doi:10.1103/PhysRevD.60.116007 hep-ph/9906555.

[24] Z. y. Duan, P. S. Rodrigues da Silva and F. Sannino, Nucl. Phys. B 592, 371 (2001) doi:10.1016/S05503213(00)00550-2 hep-ph/0001303.

[25] D. D. Dietrich, F. Sannino and K. Tuominen, Phys. Rev. D 73, 037701 (2006) doi:10.1103/PhysRevD.73.037701 hep-ph/0510217.

[26] D. D. Dietrich and F. Sannino, Phys. Rev. D 75, 085018 (2007) doi:10.1103/PhysRevD.75.085018 hep$\mathrm{ph} / 0611341$.

[27] R. Foadi, M. T. Frandsen, T. A. Ryttov and F. Sannino, Phys. Rev. D 76, 055005 (2007) doi:10.1103/PhysRevD.76.055005 arXiv:0706.1696[hep$\mathrm{ph}]$.

[28] R. Foadi, M. T. Frandsen and F. Sannino, Phys. Rev. D 87, no. 9, $095001 \quad$ (2013) doi:10.1103/PhysRevD.87.095001 arXiv:1211.1083 [hep-ph]].

[29] G. Bergner, T. A. Ryttov and F. Sannino, JHEP 1512, 054 (2015) doi:10.1007/JHEP12(2015)054 arXiv:1510.01763 [hep-th]].

[30] S. Catterall and F. Sannino, Phys. Rev. D 76, $034504 \quad$ (2007) doi:10.1103/PhysRevD.76.034504 arXiv:0705.1664 [hep-lat]].

[31] A. Hietanen, J. Rantaharju, K. Rummukainen and K. Tuominen, Nucl. Phys. A 820, 191C (2009). doi:10.1016/j.nuclphysa.2009.01.047

[32] L. Del Debbio, B. Lucini, A. Patella, C. Pica and A. Rago, Phys. Rev. D 82, 014510 (2010) doi:10.1103/PhysRevD.82.014510 arXiv:1004.3206 [heplat]].

[33] T. DeGrand, Y. Shamir and B. Svetitsky, Phys. Rev. D 83, 074507 (2011) doi:10.1103/PhysRevD.83.074507 arXiv:1102.2843 [hep-lat]].

[34] T. Appelquist, G. T. Fleming, M. F. Lin, E. T. Neil and D. A. Schaich, Phys. Rev. D 84, 054501 (2011) doi:10.1103/PhysRevD.84.054501 arXiv:1106.2148 [heplat]].

[35] T. DeGrand, Y. Shamir and B. Svetitsky, Phys. Rev. D 82, 054503 (2010) doi:10.1103/PhysRevD.82.054503 arXiv:1006.0707 [hep-lat]].

[36] Z. Fodor, K. Holland, J. Kuti, S. Mondal, D. Nogradi and C. H. Wong, JHEP 1509, 039 (2015) doi:10.1007/JHEP09(2015)039 arXiv:1506.06599 [hep- lat]].

[37] A. Hasenfratz, Y. Liu and C. Y. H. Huang, arXiv:1507.08260 [hep-lat].

[38] A. Athenodorou, E. Bennett, G. Bergner and B. Lucini, Phys. Rev. D 91, no. 11, 114508 (2015) doi:10.1103/PhysRevD.91.114508 arXiv:1412.5994 [heplat]].

[39] H. S. Fukano and F. Sannino, Phys. Rev. D 82, 035021 (2010) doi:10.1103/PhysRevD.82.035021 arXiv:1005.3340 [hep-ph]]

[40] B. Holdom, Phys. Lett. B 143, 227 (1984). doi:10.1016/0370-2693(84)90840-2

[41] G. Veneziano, Nucl. Phys. B 159, 213 (1979). doi:10.1016/0550-3213(79)90332-8

[42] P. Di Vecchia and F. Sannino, Eur. Phys. J. Plus 129, 262 (2014) doi:10.1140/epjp/i2014-14262-4 arXiv:1310.0954 [hep-ph]].

[43] E. Witten, Nucl. Phys. B 156, 269 (1979). doi:10.1016/0550-3213(79)90031-2

[44] O. Kaymakcalan, S. Rajeev and J. Schechter, Phys. Rev. D 30, 594 (1984). doi:10.1103/PhysRevD.30.594

[45] E. Witten, Phys. Lett. B 117, 324 (1982). doi:10.1016/0370-2693(82)90728-6

[46] S. Chatrchyan et al. [CMS Collaboration], JHEP 1307, 122 (2013) doi:10.1007/JHEP07(2013)122 arXiv:1305.0491 [hep-ex]].

[47] S. Fichet, G. von Gersdorff, O. Kepka, B. Lenzi, C. Royon and M. Saimpert, Phys. Rev. D 89, 114004 (2014) doi:10.1103/PhysRevD.89.114004 arXiv:1312.5153 [hep$\mathrm{ph}]]$.

[48] *** Non-standard form, no INSPIRE lookup performed $* * *$

[49] The ATLAS collaboration, ATLAS-CONF-2015-071.

[50] The ATLAS collaboration, ATLAS-CONF-2015-075.

[51] G. Aad et al. [ATLAS Collaboration], Eur. Phys. J. C 76, no. 1, 45 (2016) doi:10.1140/epjc/s10052-015-3820-z arXiv:1507.05930 [hep-ex]].

[52] G. Aad et al. [ATLAS Collaboration], JHEP 1601, 032 (2016) doi:10.1007/JHEP01(2016)032 arXiv:1509.00389 [hep-ex]].

[53] G. Aad et al. [ATLAS Collaboration], Phys. Lett. B 738, 428 (2014) doi:10.1016/j.physletb.2014.10.002 arXiv:1407.8150 [hep-ex]].

[54] CMS Collaboration [CMS Collaboration], CMS-PASB2G-13-008.

[55] G. Aad et al. [ATLAS Collaboration], JHEP 1508, 148 (2015) doi:10.1007/JHEP08(2015)148 arXiv:1505.07018 [hep-ex]].

[56] A. Pilaftsis, Phys. Rev. D (2016) 1, 015017 doi:10.1103/PhysRevD.93.015017 arXiv:1512.04931 [hep-ph]].

[57] Y. Mambrini, G. Arcadi and A. Djouadi, arXiv:1512.04913 [hep-ph]. 